\title{
Wetlands Inventory of Upper Chhokara Nalla Watershed in Chhattisgarh
}

\author{
Bharti Sahu and Jitendra Sinha* \\ Department of Soil and Water Engineering, FAE, IGKV, Raipur-492006, Chhattisgarh, India \\ *Corresponding author
}

\begin{tabular}{|l|}
\hline Ke y w o r d s \\
Wetlands, Wetlands \\
inventory, Remote \\
sensing, GIS, Upper \\
Chhokara Nalla \\
watershed, Google \\
Earth Pro \\
\hline Article Info \\
\hline $\begin{array}{l}\text { Accepted: } \\
\text { 26 January } 2021 \\
\text { Available Online: } \\
\text { 10 February } 2021\end{array}$ \\
\hline
\end{tabular}

Wetlands are ecosystems whose formation, processes and characteristics are ascertained by water. Wetlands are critically important ecosystems providing significant social, economic and ecological advantages to the mankind. The importance of wetlands in Chhattisgarh is also exemplified by the most staple food of the state "rice". Chhattisgarh is also known as "Rice bowl of India" and wetlands play an important role in the cultivation of rice. Therefore, the study has been carried out with the objective to prepare inventory of wetlands of upper Chhokara Nalla watershed (UCW). The watershed is located between $21^{\circ} 13^{\prime}$ to $21^{\circ} 14^{\prime} \mathrm{N}$ Latitude and $81^{\circ} 42^{\prime}$ to $81^{\circ} 46^{\prime} \mathrm{E}$ longitude covering an area of 1731 ha and falls in the Survey of India toposheet No. 64 G (1:250,000). The resource mapping of UCW includes Digital Elevation Model, Contour map, Drainage map etc. Wetlands inventory has been prepared from interpretation of Google Earth Pro Image in conjunction with different maps, followed by ground thruthing. The inventory includes number of wetlands, physico-chemical analysis of water samples, estimation of depth of wetlands, pre and post monsoon water spread area, estimation of volume of wetlands etc. Total numbers of 37 different wetlands have been identified inside UCW. Out of total area of wetlands (110.37 ha), river and streams occupies 42.82 ha $(38.79 \%)$, reservoirs and barrages cover 6.30 ha $(5.70 \%)$, tanks and ponds occupy 5.69 ha $(5.15 \%)$ while abandoned quarries pond have a total area 56.56 ha $(51.26 \%)$. The Percentage of wetlands inside the UCW was found to be $6.37 \%$ of the total watershed area, which is satisfactory. However, when uses are concerned, most of the wetlands are underutilized and there is potential for its judicious utilization.

\section{Introduction}

Wetland ecosystems are beauty of nature and wealth for future. These are diverse ecosystems that link people, wildlife and environment in special and interdependent ways through the essential life-support functions of water (Maltby and Barker, 2009).
Wetlands are important natural feature that modifies the human history as well as culture. Wetlands are the area of earth surface that taught the human beings the art of cultivation about 10000 years back. The wetland and human beings are in tandem from time immemorial. It is true to say that human beings are moulded and human cultures were 
modified in the vicinity of wetlands (Wade and Lopez-Gunn, 1999).

As per the Ramsar International Wetland Convention (1971) definition (Article: 1.1) wetlands are areas of marsh, fen, peat land, or water, whether natural or artificial, permanent or temporary, with water that is static or flowing, fresh, brackish or salt, including areas of marine water the depth of which at low tide does not exceed six meters. In addition, the Ramsar Convention (Article: 2.1) provides that wetlands may incorporate riparian and coastal zones adjacent to the wetlands, and islands or bodies of marine water not deeper than six meters at low tide lying within wetlands.

The information collected through wetland inventories is nowadays regarded as a necessary prerequisite for wetland conservation and management at a holistic level, involving planning on a national, regional and international scale (Dugan 1990, Hollis et al., 1992, Taylor et al., 1995, Hughes 1995, Naranjo 1995, Scott and Jones 1995). An inventory is regarded by Dugan (1990) as the first step in assembling an information base for wetland management. Strategically developed wetland inventory (or inventories) should provide managers and /or policy makers with the information base that they require not only to manage individual wetlands or threats, but to also place the conservation value of wetlands within the context of broad scale (catchment, regional or even national) land use and sustainable development priorities. Inventories are useful in the first stages of developing effective wetland conservation programs (Taylor et al., 1995, Hughes 1995, Naranjo 1995, Scott and Jones 1995, Wilen and Bates 1995). They can assist in the identification of conservation priorities, establish the basis for monitoring the ecological status of wetlands, promote awareness of wetland sites and management issues, and facilitate exchange of information and comparisons between sites and regions. As importantly, information gathered for inventories can also illustrate the economic value of wetlands and provide valuable data for resource utilisation decisions. Inventories are particularly valuable for assessing wetland loss and degradation (Taylor et al., 1995, Hughes 1995, Lu 1995, Wilen and Bates 1995). Information on rates of wetland loss and reasons for this loss have proved invaluable for promoting awareness and developing conservation and restoration programs (Hollis and Jones, 1991, Hughes 1995, Wilen and Bates 1995).

The importance of wetlands in Chhattisgarh is also exemplified by the most staple food of the state "rice". Chhattisgarh is also known as "Rice bowl of India" and wetlands play an important role in the cultivation of rice. Wetlands contribute to the economy of Chhattisgarh; firstly through agricultural production, forestry and fisheries; secondly and increasingly for water supply (for domestic use as well as for irrigation). Other economic and ecological benefits of wetlands include groundwater replenishment, maintenance of water tables for agriculture, flood control, shoreline protection and stabilisation, climate change mitigation, sediment and nutrient retention, water purification and habitats for biodiversity. Tourism in wetlands is also becoming increasingly important.

\section{Materials and Methods}

The study area upper Chhokara Nalla watershed is located between $21^{\circ} 13^{\prime}$ to $21^{\circ} 14^{\prime}$ N Latitude, and $81^{\circ} 42^{\prime}$ to $81^{\circ} 46^{\prime}$ E longitude, covers an area of 1731 ha in Raipur District Chhattisgarh as shown in fig. 1. The altitude of the watershed varies from 290 to $310 \mathrm{~m}$ above (Fig. 2 shows the method for preparation of wetlands inventory). 
Table.1 Wetlands inventory for Serikhedi pond in side of Upper Chhokranala Watershed 2016

\begin{tabular}{|c|c|c|c|c|c|c|c|}
\hline S No. & Wetlands Name & $\begin{array}{l}\text { Area } \\
\text { (ha) }\end{array}$ & $\begin{array}{l}\text { Average } \\
\operatorname{Depth}(\mathrm{m})\end{array}$ & $\begin{array}{c}\text { Storage } \\
\text { capacity }\left(\mathbf{m}^{3}\right)\end{array}$ & $\begin{array}{c}\text { Catchment } \\
\text { (ha) }\end{array}$ & Location & Climate \\
\hline & 1 & 2 & 3 & 4 & 5 & 6 & 7 \\
\hline 1 & Serikhedi Pond (A) & 2.55 & 3.5 & 89250 & 34 & $\begin{array}{l}81^{0} 43^{\prime} 39.91^{\prime \prime} \text { E Longitude } \\
21^{\circ} 13^{\prime} 59.41^{\prime \prime} \text { N Latitude }\end{array}$ & \multirow{3}{*}{ Sub-tropica } \\
\hline 2 & Serikhedi pond (B) & 1.50 & 3 & 45000 & 10 & $\begin{array}{c}81^{0} 43^{\prime} 39.91^{\prime \prime} \text { E Longitude } \\
21^{\circ} 13^{\prime} 59.41^{\prime \prime} \mathrm{N} \text { Latitude }\end{array}$ & \\
\hline 3 & Serikhadi Pond (C) & 0.34 & 3 & 10200 & 5 & $\begin{array}{l}81^{0} 43^{\prime} 5665^{\prime \prime} \text { E Longitude } \\
21^{\circ} 13^{\prime} 41.71^{\prime \prime} \text { N Latitude }\end{array}$ & \\
\hline
\end{tabular}

\begin{tabular}{|l|c|c|c|c|c|c|}
\hline Wetlands Names & $\begin{array}{c}\text { Post -Monsoon } \\
\text { (water spread } \\
\text { area) }\end{array}$ & $\begin{array}{c}\text { Per-Monsoon } \\
\text { (water spread } \\
\text { area) }\end{array}$ & Ownership & \multicolumn{2}{|c|}{ Use } & \multicolumn{2}{|c|}{ Water Quality } \\
\hline $\mathbf{1}$ & 8 & 9 & 10 & 11 & $\begin{array}{c}\text { BOD } \\
\text { ppm }\end{array}$ \\
\hline $\begin{array}{l}\text { Serikhedi Village } \\
\text { Pond(A) }\end{array}$ & 1.75 & 1.30 & Govt. & $\begin{array}{c}\text { Recharge, aquatic } \\
\text { ppm } \\
\text { life, Drinking water } \\
\text { for cattle }\end{array}$ & 7.8 & 8.9 \\
\hline $\begin{array}{l}\text { Serikhedi pond } \\
\text { (B) }\end{array}$ & 0.90 & 0.51 & Govt. & $\begin{array}{c}\text { Recharge, aquatic } \\
\text { life }\end{array}$ & 7.4 & 8.3 \\
\hline $\begin{array}{l}\text { Serikhadi Pond } \\
\text { (C) }\end{array}$ & 0.24 & - & Govt. & Recharge & 7.6 \\
\hline
\end{tabular}


Table.2 Wetlands inventory for Bhari Dam in site of Upper Chhokranala Watershed 2016

\begin{tabular}{|c|c|c|c|c|c|c|c|}
\hline S No. & Wetlands Name & $\begin{array}{l}\text { Area } \\
\text { (ha) }\end{array}$ & $\begin{array}{l}\text { Average } \\
\operatorname{Depth}(\mathrm{m})\end{array}$ & $\begin{array}{c}\text { Storage } \\
\text { capacity }\left(\mathbf{m}^{3}\right)\end{array}$ & $\begin{array}{c}\text { Catchment } \\
\text { (ha) }\end{array}$ & Location & Climate \\
\hline & 1 & 2 & 3 & 4 & 5 & 6 & 7 \\
\hline 1 & Bhari Dam & 6.30 & 3 & 189000 & 1661 & $\begin{array}{c}81^{0} 43^{\prime} 09.14^{\prime \prime} \text { E Longitude } \\
21^{\circ} 13^{\prime} 52.22^{\prime \prime} \mathrm{N} \text { Latitude }\end{array}$ & Sub-tropical \\
\hline
\end{tabular}

Continue.....

\begin{tabular}{|l|c|c|c|c|c|c|c|}
\hline Wetlands Names & $\begin{array}{c}\text { Per -Monsoon } \\
\text { (water spread } \\
\text { area) }\end{array}$ & $\begin{array}{c}\text { Post-Monsoon } \\
\text { (water spread } \\
\text { area) }\end{array}$ & Ownership & Use & \multicolumn{2}{|c|}{ Water Quality } \\
\hline $\mathbf{1}$ & 8 & 9 & 10 & 11 & 12 \\
\hline Bhari Dam & 0.13 & 4.67 & Govt. & Recharge, & BOD ppm & Turbidity ppm \\
\hline
\end{tabular}


Table.3 Wetlands inventory for Nakti Village in site of Upper Chhokranala Watershed 2016

\begin{tabular}{|c|c|c|c|c|c|c|c|}
\hline S No. & Wetlands Name & $\begin{array}{l}\text { Area } \\
\text { (ha) }\end{array}$ & $\begin{array}{c}\text { Average } \\
\text { Depth (m) }\end{array}$ & $\begin{array}{c}\text { Storage } \\
\text { capacity } \\
\left(\mathrm{m}^{3}\right)\end{array}$ & $\begin{array}{l}\text { Catchment } \\
\text { (ha) }\end{array}$ & Location & Climate \\
\hline & 1 & 2 & 3 & 4 & 5 & 6 & 7 \\
\hline 1 & Nistari Pond & 1.30 & 4 & 152000 & 101 & $\begin{array}{l}81^{0} 44^{\prime} 03.34^{\prime \prime} \text { E Longitude } \\
21^{\circ} 12^{\prime} 34.34^{\prime \prime} \mathrm{N} \text { Latitude }\end{array}$ & \\
\hline 2 & Nallah Nakti & 0.31 & 2 & 6200 & 356 & $\begin{array}{l}81^{0} 43^{\prime} 46.17^{\prime \prime} \text { E Longitude } \\
21^{\circ} 12^{\prime} 31.73^{\prime \prime} \mathrm{N} \text { Latitude }\end{array}$ & \\
\hline 3 & Nakti Abandoned Quarries -1 & 0.88 & 4 & 35200 & 113 & $\begin{array}{l}81^{0} 44^{\prime} 00.91^{\prime \prime} \text { E Longitude } \\
21^{\circ} 12^{\prime} 18.65^{\prime \prime} \mathrm{N} \text { Latitude }\end{array}$ & \multirow{7}{*}{$\begin{array}{l}\text { Sub- } \\
\text { tropical }\end{array}$} \\
\hline 4 & Nakti Abandoned Quarries -2 & 0.15 & 2 & 3000 & 111 & $\begin{array}{l}81^{0} 44^{\prime} 02.28^{\prime \prime} \text { E Longitude } \\
21^{\circ} 12^{\prime} 17.25^{\prime \prime} \mathrm{N} \text { Latitude }\end{array}$ & \\
\hline 5 & Nakti Abandoned Quarries -3 & 0.57 & 3 & 17100 & 63 & $\begin{array}{l}81^{0} 44^{\prime} 12.17^{\prime \prime} \text { E Longitude } \\
21^{\circ} 12^{\prime} 14.11^{\prime \prime} \mathrm{N} \text { Latitude }\end{array}$ & \\
\hline 6 & Nakti Abandoned Quarries -4 & 2.43 & 3 & 72900 & 64 & $\begin{array}{l}81^{0} 44^{\prime} 07.13^{\prime \prime} \text { E Longitude } \\
21^{\circ} 12^{\prime} 09.30^{\prime \prime} \mathrm{N} \text { Latitude }\end{array}$ & \\
\hline 7 & Nakti Abandoned Quarries -5 & 2.00 & 2 & 40000 & 62 & $\begin{array}{l}81^{0} 44^{\prime} 09.91^{\prime \prime} \text { E Longitude } \\
21^{\circ} 12^{\prime} 05.63^{\prime \prime} \mathrm{N} \text { Latitude }\end{array}$ & \\
\hline 8 & Nakti Abandoned Quarries -6 & 4.75 & 8 & 380000 & 30 & $\begin{array}{l}81^{0} 43^{\prime} 50.15^{\prime \prime} \text { E Longitude } \\
21^{\circ} 12^{\prime} 07.18^{\prime \prime} \mathrm{N} \text { Latitude }\end{array}$ & \\
\hline 9 & Blue Water & 4.00 & 20 & 800000 & 213 & $\begin{array}{l}81^{0} 44^{\prime} 23.88^{\prime \prime} \text { E Longitude } \\
21^{\circ} 12^{\prime} 03.80^{\prime \prime} \text { N Latitude }\end{array}$ & \\
\hline
\end{tabular}


Continue...

\begin{tabular}{|c|c|c|c|c|c|c|c|}
\hline Wetlands Name & Post-Monsoon & Pre-Monsoon & Ownership & Use & \multicolumn{3}{|c|}{ Water Quality } \\
\hline \multirow[t]{2}{*}{1} & \multirow[t]{2}{*}{8} & \multirow[t]{2}{*}{9} & \multirow[t]{2}{*}{10} & \multirow[t]{2}{*}{11} & \multicolumn{3}{|c|}{12} \\
\hline & & & & & $\mathrm{pH}$ & $\begin{array}{l}\text { BOD } \\
\text { ppm }\end{array}$ & $\begin{array}{l}\text { Turbidity } \\
\text { ppm }\end{array}$ \\
\hline Nistari Pond & 0.95 & 0.90 & Govt. & $\begin{array}{l}\text { Recharge, aquatic life, } \\
\text { Religious-Cultural activities }\end{array}$ & 7.8 & 8.5 & 9.6 \\
\hline Nall Nakti & 0.18 & 0.18 & Govt. & $\begin{array}{c}\text { Recharge Religious-Cultural } \\
\text { activities }\end{array}$ & 7.0 & 8.2 & 9.3 \\
\hline $\begin{array}{l}\text { Nakti Abandoned Quarries } \\
-1\end{array}$ & 0.42 & 0.20 & Govt. & Religious-Cultural activities & 7.1 & 8.3 & 9.4 \\
\hline $\begin{array}{l}\text { Nakti Abandoned Quarries } \\
-2\end{array}$ & 0.10 & - & Govt. & Religious-Cultural activities & 7.2 & 8.2 & 9.5 \\
\hline $\begin{array}{l}\text { Nakti Abandoned Quarries } \\
-3\end{array}$ & 0.17 & - & Govt. & Religious-Cultural activities & 7.3 & 8.4 & 9.6 \\
\hline $\begin{array}{l}\text { Nakti Abandoned Quarries } \\
-4\end{array}$ & 1.48 & 0.64 & Govt. & Religious-Cultural activities & 7.2 & 8.5 & 9.9 \\
\hline $\begin{array}{l}\text { Nakti Abandoned Quarries } \\
-5\end{array}$ & 0.33 & 0.22 & Govt. & Religious-Cultural activities & 7.0 & 8.6 & 9.7 \\
\hline $\begin{array}{l}\text { Nakti Abandoned Quarries } \\
-6\end{array}$ & 1.26 & 1 & Govt. & Religious-Cultural activities & 6.5 & 7.0 & 6.5 \\
\hline Blue water quarry & 2.91 & 2.46 & Govt. & Recreation & 6.2 & 6.5 & 6.0 \\
\hline
\end{tabular}


Table.4 Wetlands inventory for Mandir Hasod Village in site of Upper Chhokranala Watershed 2016

\begin{tabular}{|c|c|c|c|c|c|c|c|}
\hline S No. & Wetlands Name & $\begin{array}{l}\text { Area } \\
\text { (ha) }\end{array}$ & $\begin{array}{l}\text { Average } \\
\operatorname{Depth}(\mathbf{m})\end{array}$ & $\begin{array}{c}\text { Storage } \\
\operatorname{capacity}\left(\mathbf{m}^{3}\right)\end{array}$ & $\begin{array}{c}\text { Group of } \\
\text { Catchment } \\
\text { (ha) }\end{array}$ & Location & $\begin{array}{l}\text { Climat } \\
\text { e }\end{array}$ \\
\hline & 1 & 2 & 3 & 4 & 5 & 6 & 7 \\
\hline 2 & $\begin{array}{l}\text { Mandir Hashod Abandoned } \\
\text { Quarries -2 }\end{array}$ & 2.20 & 20 & 440000 & \multirow{7}{*}{225} & $\begin{array}{l}81^{0} 45^{\prime} 14.25^{\prime \prime} \text { E Long } \\
21^{\circ} 12^{\prime} 52.81^{\prime \prime} \mathrm{N} \text { Lat }\end{array}$ & \multirow{7}{*}{$\begin{array}{l}\text { Sub- } \\
\text { tropical }\end{array}$} \\
\hline 4 & $\begin{array}{l}\text { Mandir Hashod Abandoned } \\
\text { Quarries -4 }\end{array}$ & 0.30 & 20 & 60000 & & $\begin{array}{l}81^{0} 45^{\prime} 01.22^{\prime \prime} \text { E Long } \\
21^{\circ} 12^{\prime} 49.82^{\prime \prime} \mathrm{N} \text { Lat }\end{array}$ & \\
\hline 5 & $\begin{array}{l}\text { Mandir Hashod Abandoned } \\
\text { Quarries -5 }\end{array}$ & 0.40 & 20 & 80000 & & $\begin{array}{l}81^{0} 44^{\prime} 51.19^{\prime \prime} \text { E Long } \\
21^{\circ} 12^{\prime} 45.26^{\prime \prime} \mathrm{N} \text { Lat }\end{array}$ & \\
\hline 6 & $\begin{array}{l}\text { Mandir Hashod Abandoned } \\
\text { Quarries -6 }\end{array}$ & 2.35 & 20 & 470000 & & $\begin{array}{l}81^{0} 44^{\prime} 46.76^{\prime \prime} \text { E Long } \\
21^{\circ} 12^{\prime} 51.26^{\prime \prime} \mathrm{N} \text { Lat }\end{array}$ & \\
\hline 9 & $\begin{array}{l}\text { Mandir Hashod Abandoned } \\
\text { Quarries -9 }\end{array}$ & 0.94 & 20 & 188000 & & $\begin{array}{l}81^{0} 44^{\prime} 42.17^{\prime \prime} \text { E Long } \\
21^{0} 13^{\prime} 01.69^{\prime \prime} \text { N Lat }\end{array}$ & \\
\hline 10 & $\begin{array}{l}\text { Mandir Hashod Abandoned } \\
\text { Quarries -10 }\end{array}$ & 1.77 & 20 & 354000 & & $\begin{array}{l}81^{0} 44^{\prime} 46.24^{\prime \prime} \text { E Long } \\
21^{0} 13^{\prime} 02.72^{\prime \prime} \text { N Lat }\end{array}$ & \\
\hline 11 & $\begin{array}{l}\text { Mandir Hashod Abandoned } \\
\text { Quarries - } 11\end{array}$ & 1.33 & 20 & 266000 & & $\begin{array}{l}81^{0} 44^{\prime} 56.93^{\prime \prime} \text { E Long } \\
21^{0} 13^{\prime} 02.79^{\prime \prime} \mathrm{N} \text { Lat }\end{array}$ & \\
\hline
\end{tabular}




\begin{tabular}{|c|c|c|c|c|c|}
\hline 12 & $\begin{array}{l}\text { Mandir Hashod } \\
\text { Abandoned Quarries -12 }\end{array}$ & 2.85 & 20 & 570000 & $\begin{array}{l}81^{0} 45^{\prime} 03.03 " \text { E Long } \\
21^{0} 13^{\prime} 04.50^{\prime \prime} \text { N Lat }\end{array}$ \\
\hline 13 & $\begin{array}{l}\text { Mandir Hashod Abandoned } \\
\text { Quarries -13 }\end{array}$ & 3.72 & 20 & 744000 & $\begin{array}{l}81^{0} 45^{\prime} 56.03^{\prime \prime} \text { E Long } \\
21^{0} 13^{\prime} 11.81^{\prime \prime} \mathrm{N} \text { Latitude }\end{array}$ \\
\hline 14 & $\begin{array}{l}\text { Mandir Hashod Abandoned } \\
\text { Quarries -14 }\end{array}$ & 2.43 & 20 & 486000 & $\begin{array}{l}81^{0} 44^{\prime} 01.39^{\prime \prime} \text { E Long } \\
21^{0} 13^{\prime} 12.46^{\prime \prime} \mathrm{N} \text { Lat }\end{array}$ \\
\hline 15 & $\begin{array}{l}\text { Mandir Hashod Abandoned } \\
\text { Quarries - } 15\end{array}$ & 1.24 & 20 & 248000 & $\begin{array}{l}81^{0} 44^{\prime} 56.03^{\prime \prime} \text { E Long } \\
21^{0} 13^{\prime} 11.81^{\prime \prime} \mathrm{N} \text { Lat }\end{array}$ \\
\hline 16 & $\begin{array}{l}\text { Mandir Hashod Abandoned } \\
\text { Quarries -16 }\end{array}$ & 3.62 & 20 & 652000 & $\begin{array}{l}81^{0} 45^{\prime} 06.82^{\prime \prime} \text { E Long } \\
21^{0} 13^{\prime} 16.01^{\prime \prime} \mathrm{N} \text { Lat }\end{array}$ \\
\hline 17 & $\begin{array}{l}\text { Mandir Hashod Abandoned } \\
\text { Quarries -17 }\end{array}$ & 2.66 & 20 & 532000 & $\begin{array}{l}81^{0} 45^{\prime} 00.78^{\prime \prime} \text { E Long } \\
21^{0} 13^{\prime} 19.74^{\prime \prime} \text { N Lati }\end{array}$ \\
\hline 18 & $\begin{array}{l}\text { Mandir Hashod Abandoned } \\
\text { Quarries - } 18\end{array}$ & 1.85 & 20 & 370000 & $\begin{array}{l}81^{0} 45^{\prime} 00.31^{\prime \prime} \text { E Long } \\
21^{0} 13^{\prime} 24.10^{\prime \prime} \mathrm{N} \text { Lat }\end{array}$ \\
\hline 19 & $\begin{array}{l}\text { Mandir Hashod Abandoned } \\
\text { Quarries -19 }\end{array}$ & 1.35 & 20 & 270000 & $\begin{array}{l}81^{0} 45^{\prime} 02.32^{\prime \prime} \text { E Long } \\
21^{0} 13^{\prime} 28.49^{\prime \prime} \mathrm{N} \text { Lat }\end{array}$ \\
\hline 20 & $\begin{array}{l}\text { Mandir Hashod Abandoned } \\
\text { Quarries - } 20\end{array}$ & 2.57 & 20 & 514000 & $\begin{array}{l}81^{0} 45^{\prime} 03.03^{\prime \prime} \text { E Long } \\
21^{0} 13^{\prime} 04.50^{\prime \prime} \mathrm{N} \text { Lat }\end{array}$ \\
\hline 21 & $\begin{array}{l}\text { Mandir Hashod Abandoned } \\
\text { Quarries - } 21\end{array}$ & 1.34 & 20 & 268000 & $\begin{array}{l}81^{0} 45^{\prime} 56.03^{\prime \prime} \text { E Long } \\
21^{0} 13^{\prime} 11.81^{\prime \prime} \mathrm{N} \text { Latitude }\end{array}$ \\
\hline 22 & $\begin{array}{l}\text { Mandir Hashod Abandoned } \\
\text { Quarries -22 }\end{array}$ & 6.96 & 20 & 1392000 & $\begin{array}{l}81^{0} 44^{\prime} 01.39^{\prime \prime} \text { E Long } \\
21^{0} 13^{\prime} 12.46^{\prime \prime} \mathrm{N} \text { Lat }\end{array}$ \\
\hline 23 & $\begin{array}{l}\text { Mandir Hashod Abandoned } \\
\text { Quarries -23 }\end{array}$ & 5.51 & 20 & 1102000 & $\begin{array}{l}81^{0} 44^{\prime} 56.03^{\prime \prime} \text { E Long } \\
21^{0} 13^{\prime} 11.81^{\prime \prime} \mathrm{N} \text { Lat }\end{array}$ \\
\hline 24 & $\begin{array}{l}\text { Mandir Hashod Abandoned } \\
\text { Quarries - } 24\end{array}$ & 2.1 & 20 & 420000 & $\begin{array}{l}81^{0} 45^{\prime} 06.82^{\prime \prime} \text { E Long } \\
21^{0} 13^{\prime} 16.01^{\prime \prime} \mathrm{N} \text { Lat }\end{array}$ \\
\hline
\end{tabular}




\begin{tabular}{|c|c|c|c|c|c|c|c|}
\hline Wetlands Name & $\begin{array}{c}\text { Post -Monsoon } \\
\text { (water spread area)(ha) }\end{array}$ & $\begin{array}{c}\text { Pre-Monsoon } \\
(\text { water spread area)(ha) }\end{array}$ & $\begin{array}{c}\text { Ownershi } \\
\text { p }\end{array}$ & Use & \multicolumn{3}{|c|}{ Water Quality } \\
\hline \multirow[t]{2}{*}{1} & \multirow[t]{2}{*}{8} & \multirow[t]{2}{*}{9} & \multirow[t]{2}{*}{10} & \multirow[t]{2}{*}{11} & \multicolumn{3}{|c|}{12} \\
\hline & & & & & $\mathrm{pH}$ & $\begin{array}{l}\text { BOD } \\
\text { ppm }\end{array}$ & $\begin{array}{l}\text { Turbidity } \\
\text { ppm }\end{array}$ \\
\hline $\begin{array}{l}\text { Mandir Hashod } \\
\text { Abandoned Quarries -1 }\end{array}$ & 1 & 1.28 & Govt. & No use & 6.1 & 6.4 & 6 \\
\hline $\begin{array}{l}\text { Mandir Hashod } \\
\text { Abandoned Quarries -2 }\end{array}$ & 0.86 & 0.94 & Govt. & No use & 6.1 & 6.4 & 6 \\
\hline $\begin{array}{l}\text { Mandir Hashod } \\
\text { Abandoned Quarries -3 }\end{array}$ & 0.59 & 0.62 & Govt. & No use & 6.1 & 6.4 & 6 \\
\hline $\begin{array}{l}\text { Mandir Hashod } \\
\text { Abandoned Quarries -4 }\end{array}$ & 0.1 & 0.14 & Govt. & No use & 6.1 & 6.4 & 6 \\
\hline $\begin{array}{l}\text { Mandir Hashod } \\
\text { Abandoned Quarries -5 }\end{array}$ & 0.18 & 0.29 & Govt. & No use & 6.1 & 6.4 & 6 \\
\hline $\begin{array}{l}\text { Mandir Hashod } \\
\text { Abandoned Quarries -6 }\end{array}$ & 1.0 & 1.26 & Govt. & No use & 6.1 & 6.4 & 6 \\
\hline $\begin{array}{l}\text { Mandir Hashod } \\
\text { Abandoned Quarries -7 }\end{array}$ & 0.92 & 1.10 & Govt. & No use & 6.1 & 6.4 & 6 \\
\hline $\begin{array}{l}\text { Mandir Hashod } \\
\text { Abandoned Quarries -8 }\end{array}$ & 0.25 & 0.62 & Govt. & No use & 6.1 & 6.4 & 6 \\
\hline $\begin{array}{l}\text { Mandir Hashod } \\
\text { Abandoned Quarries -9 }\end{array}$ & 0.30 & 0.33 & Govt. & No use & 6.1 & 6.4 & 6 \\
\hline $\begin{array}{l}\text { Mandir Hashod } \\
\text { Abandoned Quarries -10 }\end{array}$ & 0.21 & 0.45 & Govt. & No use & 6.1 & 6.4 & 6 \\
\hline $\begin{array}{l}\text { Mandir Hashod } \\
\text { Abandoned Quarries -11 }\end{array}$ & 0.61 & 0.65 & Govt. & No use & 6.1 & 6.4 & 6 \\
\hline $\begin{array}{l}\text { Mandir Hashod } \\
\text { Abandoned Quarries -12 }\end{array}$ & 0.10 & 1.13 & Govt. & No use & 6.1 & 6.4 & 6 \\
\hline
\end{tabular}




\begin{tabular}{|c|c|c|c|c|c|c|c|}
\hline $\begin{array}{l}\text { Mandir Hashod } \\
\text { Abandoned Quarries -13 }\end{array}$ & 0.61 & 1.73 & Govt. & No use & 6.1 & 6.4 & 6 \\
\hline $\begin{array}{l}\text { Mandir Hashod } \\
\text { Abandoned Quarries -14 }\end{array}$ & 1.11 & 1.18 & Govt. & No use & 6.1 & 6.4 & 6 \\
\hline $\begin{array}{l}\text { Mandir Hashod } \\
\text { Abandoned Quarries -15 }\end{array}$ & 0.49 & 0.62 & Govt. & No use & 6.1 & 6.4 & 6 \\
\hline $\begin{array}{l}\text { Mandir Hashod } \\
\text { Abandoned Quarries -16 }\end{array}$ & 2.16 & 2.44 & Govt. & No use & 6.1 & 6.4 & 6 \\
\hline $\begin{array}{l}\text { Mandir Hashod } \\
\text { Abandoned Quarries -17 }\end{array}$ & 1.1 & 1.25 & Govt. & No use & 6.1 & 6.4 & 6 \\
\hline $\begin{array}{l}\text { Mandir Hashod } \\
\text { Abandoned Quarries -18 }\end{array}$ & 0.93 & 0.93 & Govt. & No use & 6.1 & 6.4 & 6 \\
\hline $\begin{array}{l}\text { Mandir Hashod } \\
\text { Abandoned Quarries -19 }\end{array}$ & 0.17 & 0.24 & Govt. & No use & 6.1 & 6.4 & 6 \\
\hline $\begin{array}{l}\text { Mandir Hashod } \\
\text { Abandoned Quarries -20 }\end{array}$ & 1.58 & 1.37 & Govt. & No use & 6.1 & 6.4 & 6 \\
\hline $\begin{array}{l}\text { Mandir Hashod } \\
\text { Abandoned Quarries -21 }\end{array}$ & 0.71 & 0.73 & Govt. & No use & 6.1 & 6.4 & 6 \\
\hline $\begin{array}{l}\text { Mandir Hashod } \\
\text { Abandoned Quarries -22 }\end{array}$ & 1.25 & 4.73 & Govt. & No use & 6.1 & 6.4 & 6 \\
\hline $\begin{array}{l}\text { Mandir Hashod } \\
\text { Abandoned Quarries -23 }\end{array}$ & 2.85 & 3.41 & Govt. & No use & 6.1 & 6.4 & 6 \\
\hline $\begin{array}{l}\text { Mandir Hashod } \\
\text { Abandoned Quarries -24 }\end{array}$ & - & 1.21 & Govt. & No use & 6.1 & 6.4 & 6 \\
\hline
\end{tabular}


Table 5 Wetlands inventory for Upper Chhokranala Watershed 2016

\begin{tabular}{|l|l|c|c|c|c|c|c|c|}
\hline S No. & Wetlands Name & $\begin{array}{c}\text { Number } \\
\text { of } \\
\text { streams }\end{array}$ & $\begin{array}{c}\text { Total Area } \\
\text { (ha) }\end{array}$ & $\begin{array}{c}\text { Average } \\
\text { Depth } \\
(\mathbf{m})\end{array}$ & $\begin{array}{c}\text { Length } \\
\text { (m) }\end{array}$ & $\begin{array}{c}\text { Catchment } \\
\text { (ha) }\end{array}$ & Use & Climate \\
\hline $\mathbf{1}$ & $\begin{array}{l}\text { First order } \\
\text { streams }\end{array}$ & 8 & 21.4 & 2 & 13798 & 686.4 & Irrigation \\
\hline $\mathbf{2}$ & $\begin{array}{l}\text { Second order } \\
\text { streams }\end{array}$ & 2 & 20.42 & 3 & 7685 & 1730 & Sub-tropical \\
\hline $\mathbf{3 .}$ & $\begin{array}{l}\text { Third streams } \\
\text { order }\end{array}$ & 1 & 1.00 & 4 & 0.33 & 1731 & Irrigation \\
\hline
\end{tabular}


Table.6 Area estimates of Wetland in Upper Chhokranala Watershed (2016)

\begin{tabular}{|c|c|c|c|c|}
\hline S. No & Wetland Category & $\begin{array}{l}\text { Number of } \\
\text { wetlands }\end{array}$ & $\begin{array}{l}\text { Total Wetland } \\
\text { Area }\end{array}$ & $\begin{array}{l}\% \text { of wetland } \\
\text { area }\end{array}$ \\
\hline \multicolumn{5}{|c|}{ Inland Wetland-Natural } \\
\hline 1 & Lakes/ Ponds & - & - & - \\
\hline 2 & $\begin{array}{l}\text { Ox-bow lakes/cut off } \\
\text { meanders }\end{array}$ & - & - & - \\
\hline 3 & Riverine Wetlands & - & - & - \\
\hline 4 & Waterlogged & - & - & - \\
\hline 5 & River/stream & $1 *$ & 42.82 & 38.79 \\
\hline 6 & Karst & - & - & - \\
\hline 7 & High altitude wetland & - & - & - \\
\hline \multicolumn{5}{|c|}{ Inland Wetland-Man-made } \\
\hline 7 & Reservoirs/Barrages & 1 & 6.30 & 5.70 \\
\hline 8 & Tanks/Ponds & 4 & 5.69 & 5.15 \\
\hline 9 & Waterlogged & - & - & - \\
\hline 10 & Salt pan & - & - & - \\
\hline 11 & Abandoned Quarries & 31 & 56.56 & 51.24 \\
\hline \multicolumn{2}{|c|}{ Sub total } & 37 & 110.37 & 100 \\
\hline \multicolumn{3}{|c|}{ Total Area of watershed } & \multicolumn{2}{|c|}{1731 ha } \\
\hline
\end{tabular}


Fig.1 Location Map of the Study area
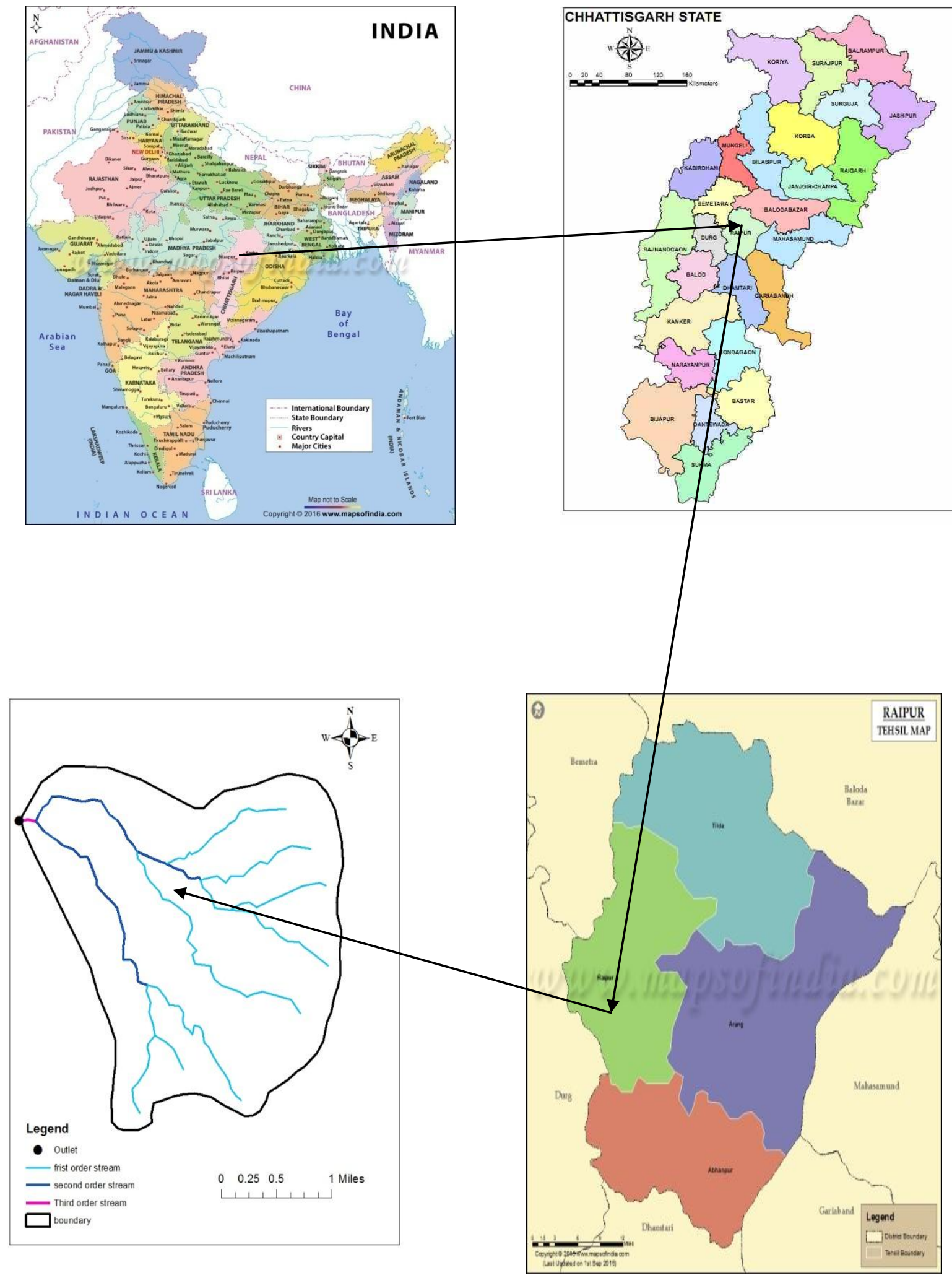
Fig.2 Methodology for preparation of wetlands inventory

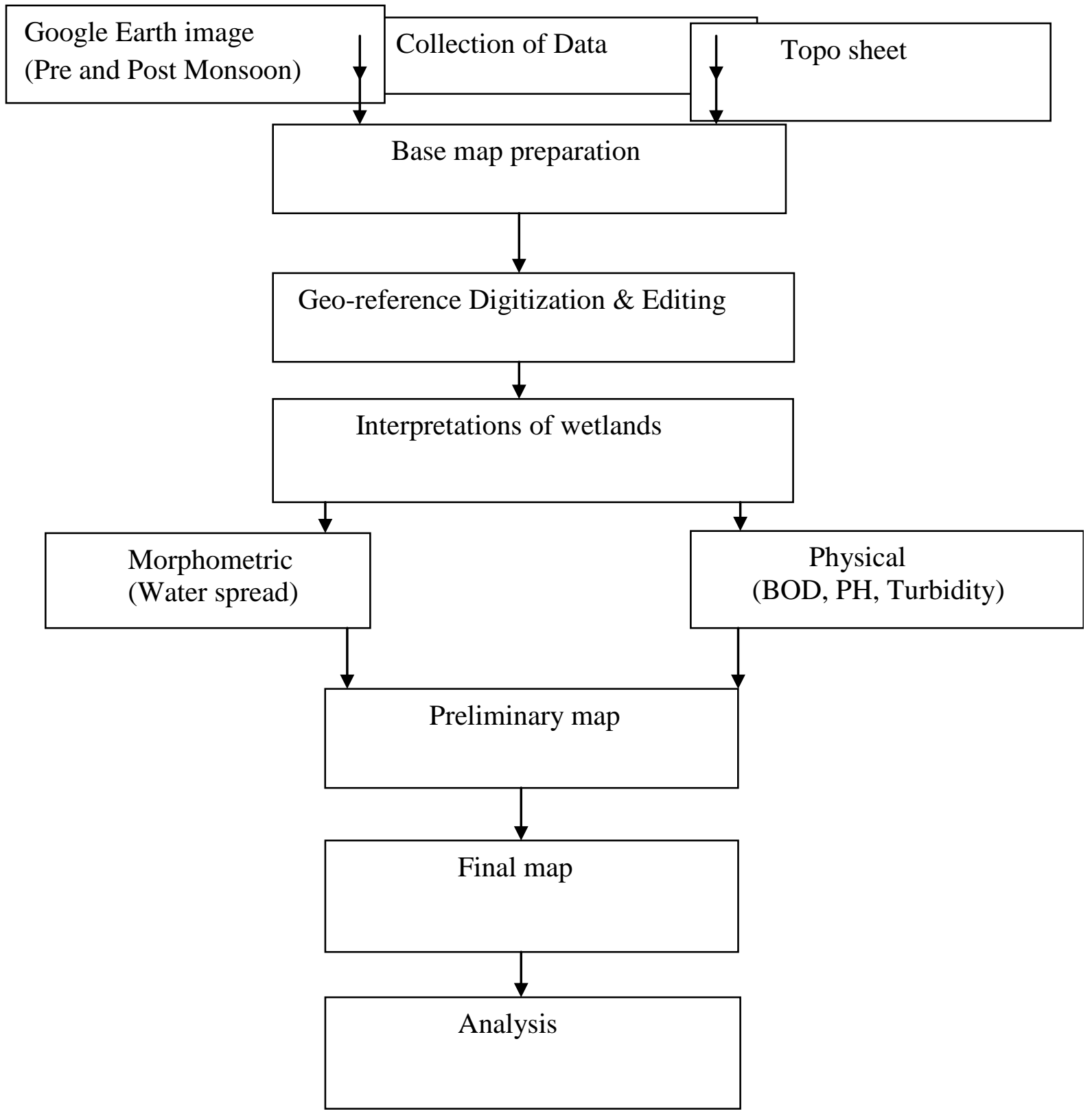


Fig.3 Total Wetlands area inside Upper Chhokranalla Watershed

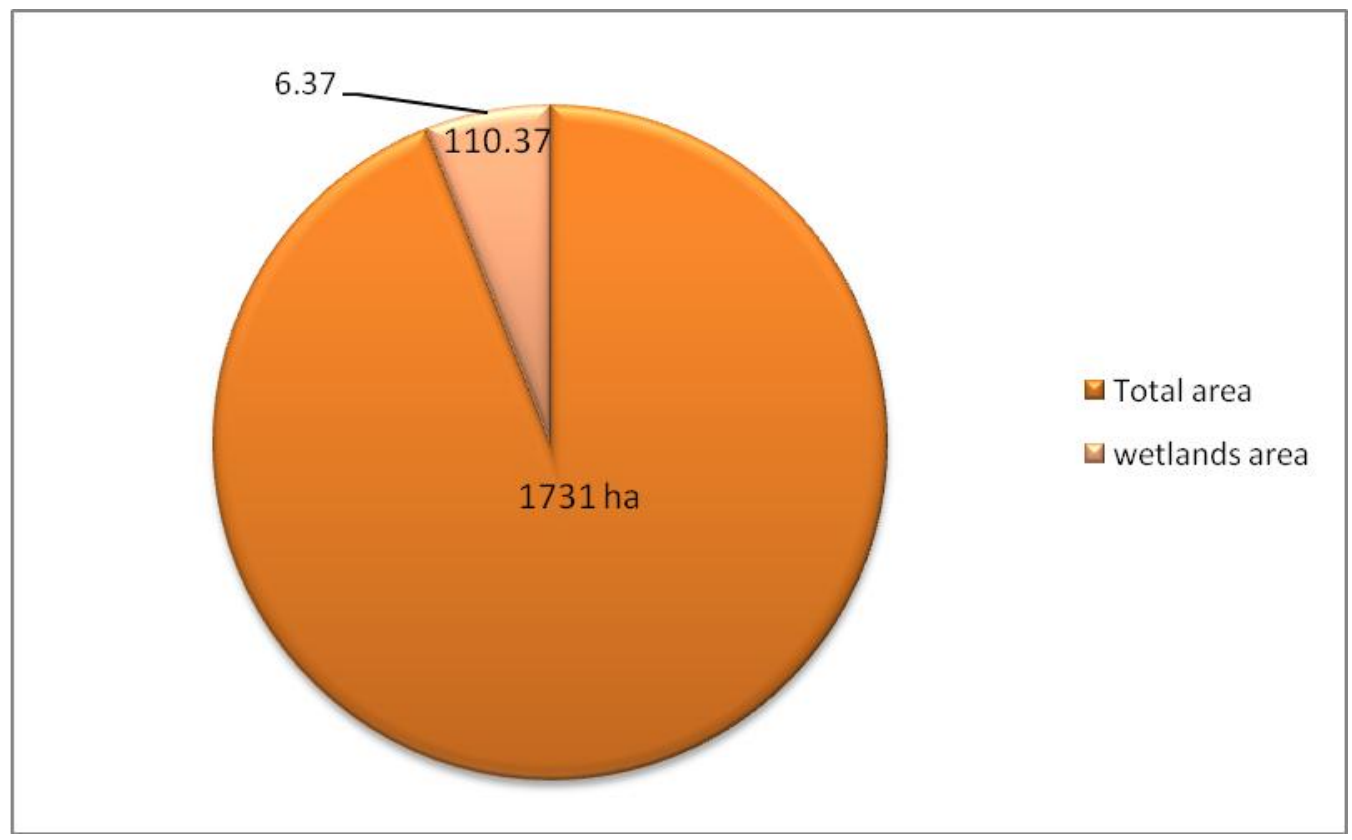

Fig.4 Type wise distribution of wetlands in Upper Chhokranalla Watershed

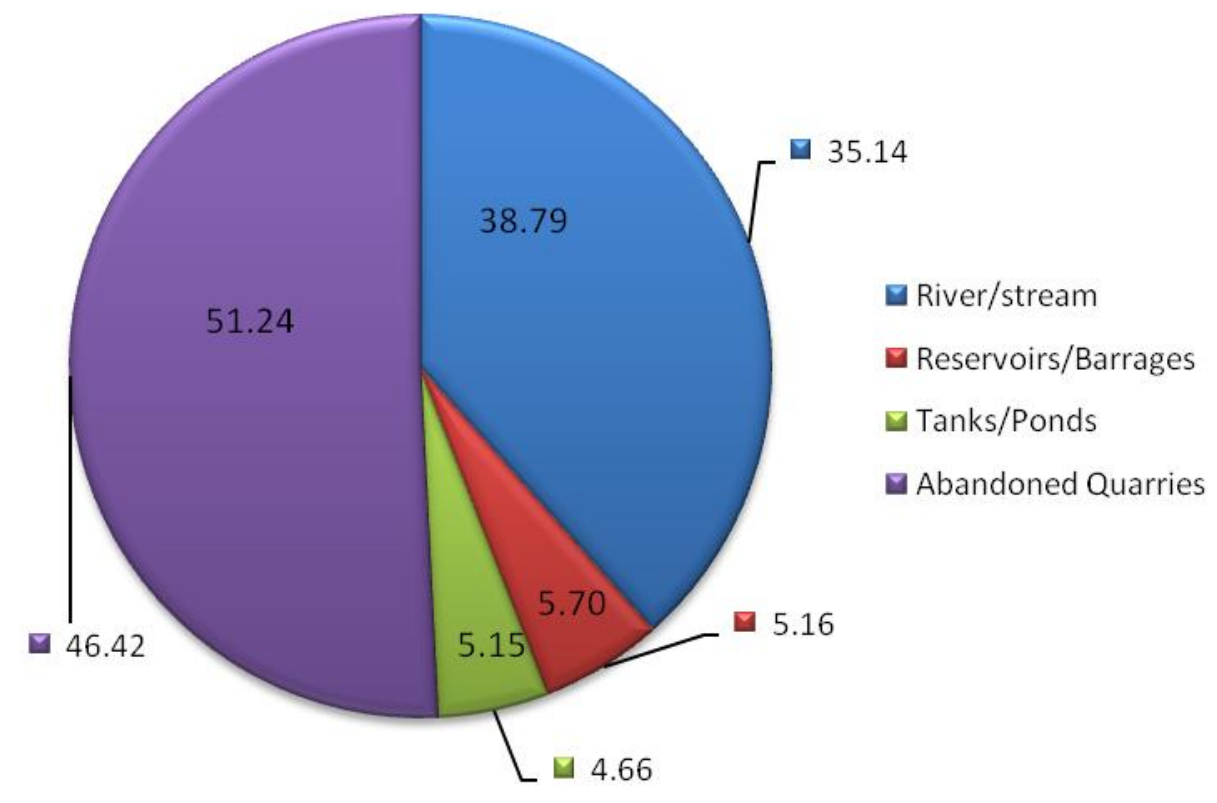


Fig.5 Wetlands identified in side Upper Chhokranalla Watershed 2016 (Source: Google Earth Image)

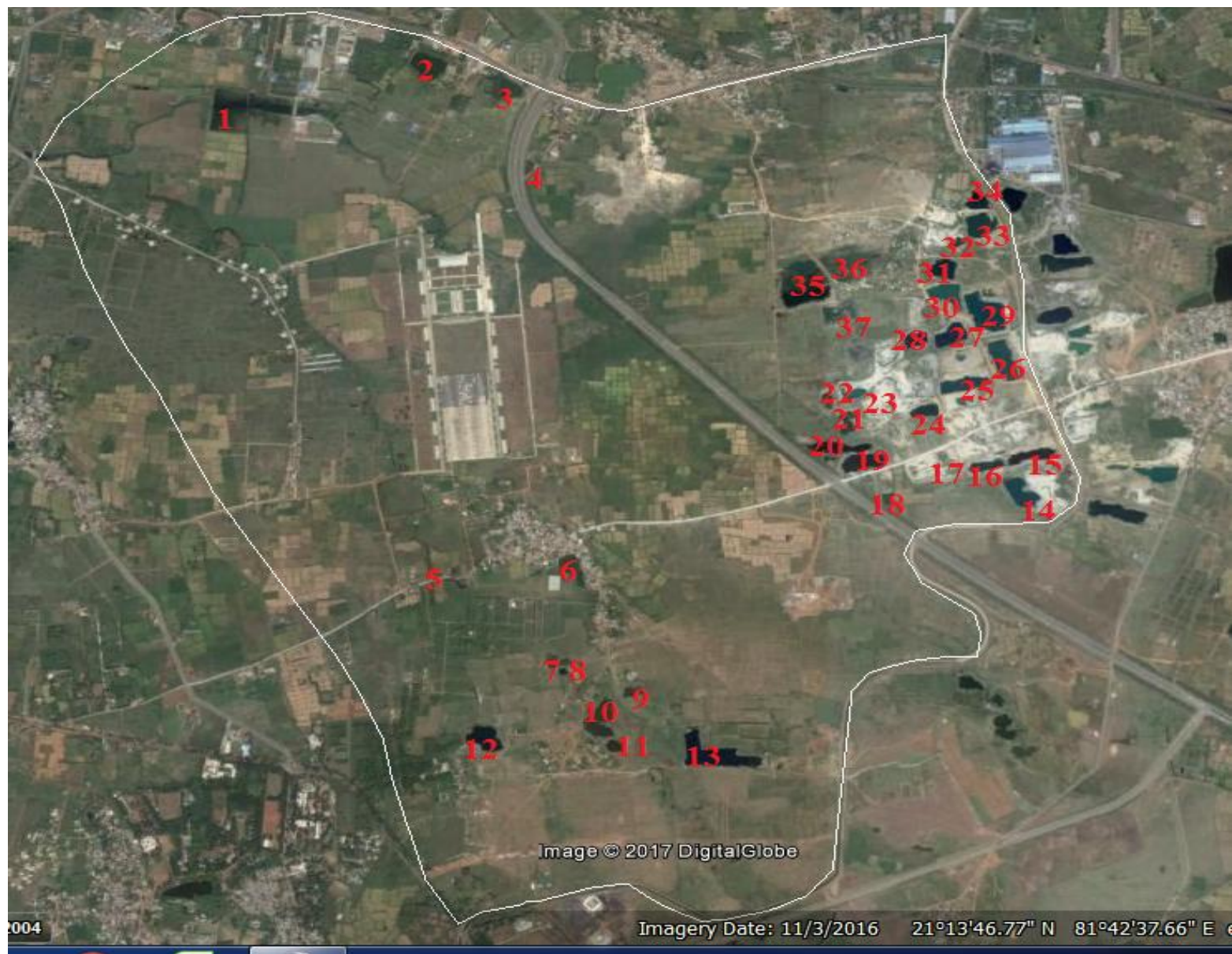

\section{Results and Discussion}

Field survey and Google Earth image interpretation work was carried out to document and inventorize the wetlands of Upper Chhokara Nalla Watershed. Total numbers of Thirty Seven different wetlands have been identified in the Upper Chhokara Nalla Watershed. These Wetlands are marked in the UCW map (Fig. 5) and are given in Table $1-6$. There are 37 wetlands (110.37 ha) inside the catchment area of 1731 ha in the Upper Chhokara Nalla Watershed. River and streams occupied $38.79 \%$ of the wetlands, reservoirs and barrages cover 5.70 ha, and tanks and ponds 5.15 ha have a total area of 6.30 ha. Abandoned Quarries ponds have a total area 56.6 ha. Details of estimates of wetlands in Chhokara Nalla Watershed are given in Table 6 and fig. 3 and 4.

In conclusions, percentage of wetlands inside the Upper Chhokara Nalla Watershed was found to be $6.37 \%$ of the total area, which in satisfactory. However, when uses are concerned, most of the wetlands are underutilized and there is potential for its judicious utilization.

\section{References}

Dugan PJ 1990. Wetland conservation: A review of current issues and required action. IUCN, Gland, Switzerland. 
Hollis GE, Patterson J, Papayannis $\mathrm{T}$ and Finlayson CM (1992). Sustaining wetlands Policies, programs and partnerships. In Managing Mediterranean wetlands and their birds, IWRB Special Publication No. 20, Slimbridge, UK, 281-285.

Hughes JMR (1995). The current status of European wetland inventories and classifications. In Classification and inventory of the world's wetlands, eds $\mathrm{CM}$ Finlayson and $\mathrm{AG}$ van derValk, Advances in Vegetation Science 16, Kluwer Academic Publishers, Dordrecht, The Netherlands, 17-28.

Naranjo LG 1995. An evaluation of the first inventory of South American wetlands. In Classification and inventory of the world's wetlands, Advances in Vegetation Science 16, Kluwer Academic Publishers, Dordrecht, The Netherlands, 125-129

National wetlands Atlas, Space Applications Centre (ISRO), Ahmadabad Ministry of Environment and Forests, Government of India March 2011.

Maltby, E. and Barker, T.,2009. The wetlands Handbook.1st ed. (Oxford: Blackwell Publishing)

Ramsar, (2002), Wetlands, water, life, and culture, 8th meeting of the conference of contracting parties to the convention of wetlands (Ramsar, Iran, 1971).

Ramsar, (2010), wetlands inventory handbooks for the wise use of wetlands 4th edition.

Scott DA and Jones TA 1995. Classification and inventory of wetlands: A global overview. In Classification and Inventory of the World's Wetlands, Advances in Vegetation Science 16, Kluwer Academic Publishers, Dordrecht, The Netherlands.

Taylor ARD, Howard GW and Begg GW 1995. Developing wetland inventories in southern Africa: A review. In Classification and inventory of the world's wetlands, eds CM Finlayson and $\mathrm{AG}$ van der Valk, Advances in Vegetation Science 16, Kluwer Academic Publishers, Dordrecht, The Netherlands, 57-79.

Wade, M., and Lopez-Gunn, E., (1999) Wetland conservation, in Pacione, $\mathrm{M}$. ,ed., Applied Geography Principles and Practice, Rout ledge, p. 632.

Wilen BO and Bates MK 1995. The US Fish and Wildlife Service's national wetland inventory project. In Classification and inventory of the world's wetlands, eds CM Finlayson and AG vander Valk, Advances in Vegetation Science 16, Kluwer Academic Publishers, Dordrecht, The Netherlands, 153-169.

\section{How to cite this article:}

Bharti Sahu and Jitendra Sinha. 2021. Wetlands Inventory of Upper Chhokara Nalla Watershed in Chhattisgarh. Int.J.Curr.Microbiol.App.Sci. 10(02): 3302-3318.

doi: https://doi.org/10.20546/ijcmas.2021.1002.363 